\title{
Síndrome de aplastamiento subclavio. Ruptura completa de electrodo de marcapaso
}

\author{
Subclavian crush syndrome. Complete rupture of pacemaker electrode
}

\author{
Rafael Espinosa Fernández*
}

Citar como: Espinosa FR. Síndrome de aplastamiento subclavio. Ruptura completa de electrodo de marcapaso. Acta Med Grupo Angeles. 2021; 19 (2): 294-295. https://dx.doi.org/10.35366/100460

Una mujer de 77 años de edad acudió a consulta de cardiología por estados presincopales, diaforesis profusa y aisladas palpitaciones. En el electrocardiograma, se observó fibrilación auricular con frecuencia ventricular media de $45 x^{\prime}$ y pausas repetidas mayores de 2.5 segundos de duración. Se indica implantar marcapaso VVI a través de punción de la vena subclavia derecha, utilizando la técnica estándar.

Durante las revisiones rutinarias posteriores del marcapaso, se registraban impedancias de 640 ohmios (ohm). Dos años después del implante, en la consulta de cardiología, refirió mareos ocasionales. En el registro de los parámetros eléctricos del marcapaso se detectó una impedancia mayor de 2,500 ohm. La radiografía de tórax mostró ruptura completa del electrodo por delante de la clavícula (Figura 1).

\section{DISCUSIÓN}

Para el implante del marcapaso se utiliza la punción de la vena subclavia. Este abordaje es seguro y con baja frecuencia de complicaciones. Uno de los tropiezos más importantes de esta técnica es la fractura del electrodo por deslizamiento en la región costoclavicular, por delante del sitio de incisión. ${ }^{1-3}$

Otra zona relacionada con tales quebrantos es el área de fijación del electrodo, cercano al marcapaso por fuera del sistema vascular.

Figura 1:

Radiografía del tórax muestra sección completa del electrodo del marcapaso en borde inferior de la clavícula.
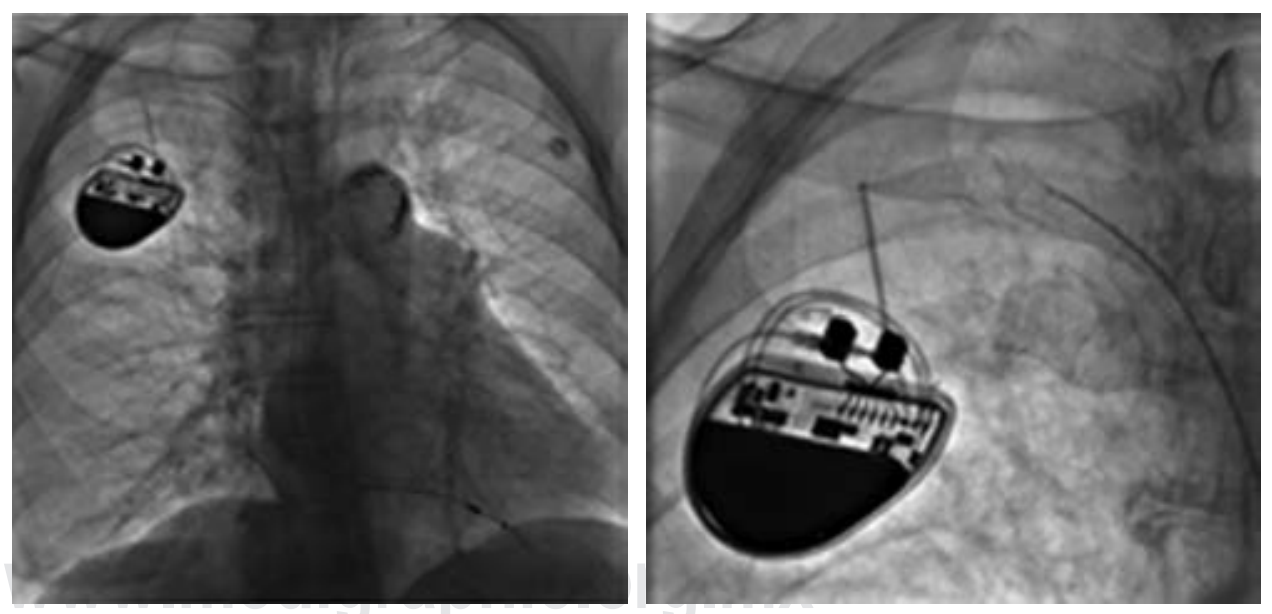

* Servicio de Cardiología. Hospital Ángeles Lomas, Estado de México.

Aceptado: 05-05-2020.

Correspondencia:

Rafael Espinosa Fernández

Correo electrónico: respinosacardiogia@hotmail.com www.medigraphic.com/actamedica 
El síndrome de aplastamiento subclavio es la fractura del mencionado componente, secundaria a la fricción del elemento, que se ejerce entre la clavícula y la primera costilla después de la punción en la vena subclavia. ${ }^{4,5}$

La prevalencia de semejante evento es del 1.0\% al 7\% dentro del sistema vascular, dependiendo de las series publicadas. ${ }^{1-3}$

Una de las consecuencias graves de tal rotura es la embolia pulmonar de la porción distal del electrodo, como se ha reportado en algunos casos. ${ }^{6,7}$

El implante del electrodo a través de la disección de la vena cefálica tiene menos posibilidades de esta complicación, a diferencia de la punción de la subclavia.

La medición de la impedancia, que es la resistencia al paso de la corriente eléctrica a través del electrodo, es un dato muy importante para conocer la integridad de dicho elemento. En este caso, en forma inesperada se observó elevación muy importante de las resistencias eléctricas con pocos síntomas. La radiografía del tórax corrobora el diagnóstico.

En las revisiones de los parámetros eléctricos de los marcapasos es imprescindible registrar en forma cuidadosa la impedancia, como otra métrica en la sospecha de fractura del electrodo, a pesar de que los pacientes no tengan síntomas.

\section{REFERENCIAS}

1. Gallik DM, Ben-Zur UM, Gross JN, Furman S. Lead fracture in cephalic versus subclavian approach with transvenous implantable cardioverter defibrillator systems. Pacing Clin Electrophysiol. 1996; 19 (7): 1089-1094.

2. Kazama S, Nishhiyama K, Machii M, Tanaka K, Amano T, Nomoura $K$ et al. Long-term follow up of ventricular endocardial pacing leads: complications, electrical performance, and longevity of 561 ventricular leads. Jpn Heart J. 1993; 34 (2): 193-200.

3. Jacobs DM, Fink AS, Miller RP, Anderson WR, McVenes RD, Lessar JF, Cobian KE et al. Anatomical and morphological evaluation of pacemaker lead compression. Pacing Clin Electrophysiol. 1993; 16 (3 Pt 1): 434-444.

4. Weiner S, Patel J, Jadonath RL, Goldner BG, Gross JN. Lead failure due to the subclavian crush syndrome in a patient implanted with both standard and thin bipolar spiral wound leads. Pacing Clin Electrophysiol. 1999; 22 (6 Pt 1): 975-976.

5. Vyselaar JR, Michael KA, Nolan RL, Baranchuk A. Left subclavian vein occlusion after pacemaker insertion. Cardiovasc J Afr. 2008; 19 (3): 155.

6. Theiss W, Wirtzfeld A. Pulmonary embolization of retrained transvenous pacemarker electrode. Br Heart J. 1976; 38: 326-330.

7. Said SA, Ticheler CH, Stassen CM, Derks A, Droste HT. Possible complications of subclavian crush syndrome. Neth Heart J. 2005; 13 (3): 92-97. 